\title{
Novel Developments in Polidocanol Sclerotherapy: A Review
}

\author{
Xiang Zheng, Qiming Wei, Hong Zhang* \\ Department of Interventional Radiology and Vascular Surgery, The First Affiliated Hospital of Jinan University, \\ Guangzhou, China \\ Email:*elsker2000@sina.com
}

How to cite this paper: Zheng, X., Wei, Q.M. and Zhang, H. (2018) Novel Developments in Polidocanol Sclerotherapy: A Review. Journal of Biosciences and Medicines, 6, 31-41.

https://doi.org/10.4236/jbm.2018.68003

Received: June 4, 2018

Accepted: August 3, 2018

Published: August 6, 2018

Copyright $\odot 2018$ by authors and Scientific Research Publishing Inc. This work is licensed under the Creative Commons Attribution International License (CC BY 4.0).

http://creativecommons.org/licenses/by/4.0/

\section{(c) (i) Open Access}

\begin{abstract}
Polidocanol is the most widely used Sclerotherapy in European and American countries. It is affordable, easy to use and apply, and has high efficiency. The purpose of this paper is to give an overview of the recent basics of the Sclerotherapy, chemistry and formulation, clinical applications, and side-effects and complications of Polidocanol, and to discuss the Polidocanol as the safety and efficacy of Sclerotherapy.
\end{abstract}

\section{Keywords}

Polidocanol, Sclerosant, Sclerotherapy, Foam

\section{Introduction}

Polidocanol is the most widely used sclerosant in Sclerotherapy in western countries. It can be applied on varicose veins, esophageal variceal, vascular malformations, hemangiomas, internal hemorrhoids and cystic diseases. Chemical Sclerotherapy has been performed for over 75 years [1]. The direct intravascular injection of liquid or foamed agent stimulates [2] [3] endothelial cells damage of target vessels and then stimulates transmural vessel walls. Thrombus formation occurs locally, and the damaged veins are eventually transformed into a fibrous cord, which subsequently cannot be recanalized [4]. The safety of Polidocanol has been recognized by the international medical community as [1]. So far, the only drug approved for Sclerotherapy has been used in Europe. Polidocanol is also approved by the Food and Drug Administration of the United States in March, 2010. As a new type of foam hardening, the clinical application of these agents will be more and more extensive, and we should concentrate more on them. 


\section{Introduction to the Compound}

Polidocanol is a synthetic fatty alcohol, that consists of $95 \%$ hydroxypolyethoxydodecane and $5 \%$ ethyl alcohol. It is a nonionic surfactant whose chemical structure forms an alkyl chain of $12 \sim 14$ carbon atoms $\left(\mathrm{C}_{12}-\mathrm{C}_{14}\right)$ and an ethylene oxide chain of 9 ethylene oxide units. Its average empirical formula is $\left[\mathrm{C}_{30} \mathrm{H}_{62} \mathrm{O}_{10}\right]$. It is a viscous liquid at the room temperature, having a melting point of $15^{\circ} \mathrm{C} \sim 21^{\circ} \mathrm{C}$. It is miscible in water, having $\mathrm{pH}$ value of $6 \sim 8$ (water $1 \%$ ), and having a density of $0.97 \mathrm{~g} / \mathrm{cm}^{3}$ at room temperature, which is close to water [5] [6].

Polidocanol was first developed in 1931 and was originally used as local anesthetic. It has been shown not to have any local anesthetic property because of lack of an aromatic ring. In 1963, Henschel found out that intravascular injection of high concentration of Polidocanol caused stimulation of the vascular wall. For the first time, the effect of Polidocanol on the treatment of Sclerotherapy [7] in general foam Sclerotherapy did not become popular until the 2000's, by which time efficient methods for transforming liquid sclerosing solutions into foam were well described by Tessari [8].

\section{Chemistry and Formulation}

\subsection{Mechanism of Sclerosing Action}

Polidocanol shows its sclerosant effects by causing concentration dependent differential cell injury [9]. Cellular calcium signaling and nitric oxide pathways become activated by the administration of the sclerosant, followed by cell death. Intravascular and intravascular injection of Polidocanol can cause direct damage to vascular endothelium, and then provoke formation of thrombus, being adhesion to the intravascular injection site. Then produce inflammatory lesions and tissue fibrosis, fibrotic cord is instead of pathological blood vessels, leading to the permanent occlusion of pathological vessels, so as to achieve the aim of sclerosis [10].

\subsection{Foam Polidocanol Formulation}

In recent years, the use of foamed sclerosants is becoming more and more popular. Single layer endothelial cells and animal experiments show that foam sclerosing agents could reach the effective concentration of endothelial cells rather than liquid sclerosing agent. This stronger effect stems from the fact that foam hardener can squeeze blood out and act directly on the walls of blood vessels. Also, it is harder to be diluted with blood for longer periods. In general, foam sclerosing agent is at least twice as effective as the liquid sclerosing agent in the same concentration and volume [11] [12]. The effect of sclerosis depends on foam quality; an unstable foam needs a higher quantity of liquid sclerosant for the same effect as a stable foam. By controlling the stability of foam, one can control the duration of its contact with the blood vessel and therefore the effect of the sclerosing agent [5] [10] [12] [13]. 
A lot of methods and mechanisms have been used to mix and agitate liquid sclerosants with gas admixtures to create foams for clinical use. Based on the technique initially by Tessari, making of foam is easy and quick: it can be prepared with syringes, a three-way valve, drugs and air/gas. More and more people use the following method: inhaling 1 cubic centimeter sclerosing agent in one syringe, the other by inhaling 4 cubic centimeter filter air, connecting to a cock valve, applying a syringe to another injector through one syringe to another injector, and 5 cubic centimeter foam is formed [8]. Such foams commonly employ a ratio of gas to liquid ranging from 1:1 to 8:1, producing foams of vary densities and rheological properties [14]. The concentration of sclerosing agent, the temperature of foam formation, the type and composition of gas foam, the characteristics of syringes and needles (materials) can affect the quality of the foam and affect the treatment effect [15].

In the United States, Varithena is the only commercially approved ready-made injectable sclerosing foam, which is composed of Polidocanol and gas 65\% oxygen and $35 \%$ carbon dioxide by using a proprietary pressurized canister mechanism [16]. In particular, the Varithena ${ }^{\oplus}$ foam is appreciably smaller than that from manual foam production techniques, with reproducible rheological properties and more rapid absorption of bubbles within the body [2] [17].

\section{Clinical Application}

\subsection{Polidocanol Sclerotherapy for Varicose Veins}

Varicose veins of the lower extremities are common in adults. A study shows that nearly $30 \%$ of the population has visible diseases with varicose veins or nutritional changes in the lower extremities, and $28 \%$ had functional diseases of the superficial or deep veins of the lower extremities [18]. Beyond the direct visibility of abnormal visible veins, patients can suffer pain, alterations in skin color, inflammation, induration and skin ulceration [19]. Foam Sclerotherapy is a relatively new minimally invasive endovenous technology, which has replaced open surgery in many clinics. Direct diluted foam Sclerotherapy (or direct liquid Sclerotherapy), according to the degree of varicose veins, point to point injection, foam hardening agent at each point $1 \sim 2 \mathrm{~mL}$ until the varicose veins become sclerosis or thrombosis [20]. The total amount of each treatment is calculated by liquid, not exceeding $8 \mathrm{ml}$. The foam hardening of the main stem needs to be conducted under ultrasound guidance, high ligation or main vein stripping of great saphenous vein [21]. In a study of 338 cases of varicose veins, the treatment success rate of foam Polidocanol at 12 weeks was $96 \%$ and $95 \%$ at 26 weeks [22]. A 5 years follow-up clinical trial of 196 patients comparing the open surgery and the foam sclerosing agent for the varicose veins of the great saphenous vein, the effective rate of the open operation group is $96 \%$, and that of foam sclerosing agent is $64 \%$ [23]. The side effects and complications of sclerosing therapy are rare. The most common side effects are heme deposition and hyperpigmentation around the blood vessels, which occur in about $50 \%$ of the patients [24]. 


\subsection{Polidocanol Sclerotherapy for Esophageal Variceal}

Esophageal variceal bleeding is a life-threatening complication of liver cirrhosis. It has a high probability of recurrence and is associated with a significant morbidity and mortality. Endoscopic injection Sclerotherapy has been gradually applied to the management of esophageal variceal bleeding since the mid-1970s and is effective in the control of acute bleeding, which decreases the rate of re-bleeding and prolongs the survival time. At present, endoscopic variceal ligation and endoscopic Sclerotherapy are the first line treatments for acute gastroesophageal variceal bleeding [25]. Intravascular and paravascular injection of Polidocanol, which can eradicate varices and collateral vessels, causes the fibrosis of the lower esophageal submucosal tissue, thus decreasing recurrence of varices [26]. A Prospective Study of Endoscopic Injection Polidocanol and Endoscopic Variceal Ligation in the Treatment of Esophageal Varices in 2017 concluded that both band ligation and Sclerotherapy are safe and effective, no difference in mortality rate and Sclerotherapy is considered as the first-line treatment for bleeding cases [27]. However, the incidence of complications of endoscopic injection Polidocanol is up to $20 \%$, such as adverse pulmonary and renal effects, esophageal ulceration, stricture and perforation [28]. With the development of the latest endoscopic Sclerotherapy, the risk of serious side effects, such as perforation of esophagus, ectopic embolism and hemorrhagic ulcers, has been reduced in using Polidocanol.

\subsection{Polidocanol Sclerotherapy for Hemangioma and Vascular Malformation}

Hemangioma (HMS) is a vascular tumor or vascular anomaly caused by vascular hyperplasia, and vascular malformation (VMS) is the result of the stagnation of the venous system during the development of the embryo [29]. In recent years, ultrasound-guided Sclerotherapy with foam has been proven as an alternative treatment for hemangiomas and vascular malformations, especially when complete resection cannot be performed in surgical treatment or the situation is too damage. Polidocanol has less sclerosis power than that of ethanol, but with better tolerance [30]. Sclerotherapy for hemangiomas and vascular malformations can be performed in superficial and intramuscular lesions. Multi point multiple Sclerotherapy has achieved good clinical treatment. A meta-analysis is conducted in a total of 19 randomized controlled trials (RCTs) involving 1514 participants. In conclusion, Polidocanol is an effective and safe therapy for HMs and VMs (especially venous malformations) [31].

Europe Guideline on Sclerotherapy is recommended that foam form is better than liquid form for the treatment of venous malformations in 2013 [32]. Blaise et al demonstrated the effect of ultrasound-guided Sclerotherapy with foam Polidocanol for the treatment of venous malformations in 25 patients between 7 and 78 years old. It used $0.25 \%$ - 3\% foam Polidocanol, the number of sessions needed was 2.3 ranging between 1 and 16 sessions [33]. After follow-up for five 
months, the size of lesion and pain in 23 patients are reduced. There are over $50 \%$ reductions in 9 patients and below $50 \%$ in 14 patients. Only 2 patients have serious side effects. In addition, it also has the anesthetic effect for pain relief at injection site. The combination therapy of Polidocanol with other treatments will produce excellent responses.

\subsection{Polidocanol Sclerotherapy for Hemorrhoids}

Hemorrhoids are engorgement of vascular cushions lining the anal canal. Almost half of over 50 years old individuals have hemorrhoids. The most common presentation is bright red and painless rectal bleeding during defecation [34]. Polidocanol is the only approved medication for Sclerotherapy in first and second-degree hemorrhoid [35] in Germany. Polidocanol induces a series of inflammatory reaction, leading to local sclerosis of the submucosal tissue and consequently in fixation of the hemorrhoidal tissue to the underlying tissue. In addition, the obliteration of the vascular bed in and around the hemorrhoid may lead to shrinkage. Foam and liquid Polidocanol show a good safety profile. Foam Polidocanol is a new, innovative and highly effective method. In a randomized, controlled, single-blinded and multicenter clinical trial published in 2013, efficacy and safety of Sclerotherapy with foam Polidocanol is proved in comparison with liquid Polidocanol in the treatment of first-degree hemorrhoid. After one Sclerotherapy session, success rate was $88 \%$ with Polidocanol foam and $69 \%$ with fluid Polidocanol, no serious adverse events at all [36]. Sclerotherapy is a very safe treatment option, but the most common malaise is pain at injection site during or after injection, which can last for several hours at most. Appropriate injection techniques and optimal injection volume of Sclerotherapy are necessary conditions to avoid complications.

\subsection{Polidocanol Sclerotherapy for Cystic Diseases}

It is reported that Polidocanol is used as a sclerosing agent for Sclerotherapy in hepatic and renal cysts for the first time in 1990 [37]. With the development of imaging and medical technology, interventional minimally invasive treatment of cystic disease under imaging guidance has been widely used in clinical practice. Compared with open and endoscopic surgery, percutaneous Sclerotherapy can achieve the same effect as surgical laparotomy under minimally invasive conditions due to its minimal trauma, low patient suffering and low cost, thus, gradually changing from the original status of adjuvant therapy to a dominant position. The application of percutaneous drainage and Polidocanol sclerosis in cystic diseases including congenital "true" splenic cysts, benign thyroid cysts, hydrocele, epididymal cysts and prostate cysts is considered as a simple low-cost surgical treatment alternative [38] [39]. Ormeci's study reported that Polidocanol is also used as sclerosant in the treatment of hepatic hydatid cysts, splenic hydatid cysts, kidney hydatid cysts, and muscle hydatid cysts with satisfactory long-term results [40]. Rastogi reported that Polidocanol as a sclerosing agents 
can treat aneurysm and bone cysts under fluoroscopic guidance and use as general anesthesia, sedation or local anesthesia [41]. Recent studies have found that Polidocanol Sclerotherapy for the treatment of digital myxoid cysts and postoperative lymphatic cysts are considered to be a promising, effective and well-tolerated new treatment [42].

\subsection{Polidocanol Sclerotherapy for Chronic Tendinitis}

Early studies show that the area of neovascularization (neovascularization and nerves) maybe the cause of chronic Achilles tendinitis [43]. Topical administration of sclerosing drugs (Polidocanol) may relieve pain [44]. In the process of Polidocanol being used increasingly for the treatment of chronic Achilles tendinitis, a few inconsistent findings have emerged. There is a case of a complete rupture of achilles tendon in a 78-year-old woman after injection of Polidocanol [45]. Sclerotherapy with Polidocanol for neovascularization of patellar tendinosis may relieve tendon pain and recover normal tibial tendon load activity. It is reported that the treatment of chronic pain in tennis elbow (lateral epicondylitis) using injection of hard Polidocanol may reduce tendon pain and increase grip strength [46]. However, a meta-analysis shows that there is no significant change in use of Polidocanol compared with that of placebo [47]. It is reported that patients suffer chronic painful shoulder impingement syndrome after injection of Polidocanol. In the short-term efficacy, it seems that the injection of sclerotic Polidocanol for supraspinatus muscle tendon and/or cystic wall neovascularization may reduce the amount of shoulder pain during loading events [48].

\subsection{Polidocanol Sclerotherapy for Other}

Preoperative portal vein embolization can induce residual liver hypertrophy and atrophy at the same time. This procedure has been used to avoid liver failure in extensive hepatic resection recently. It is reported that when the Polidocanol and gelatin sponge are used in embolization of the portal vein, then obstruction of the portal vein is maintained. It induces sufficient hypertrophy and was the suitable agent for portal embolization [49].

Foam Polidocanol (PF) has been studied as a safe and cheap method for permanent contraception compared with surgical permanent contraception for women recently [50]. In addition, Polidocanol injection decreases the bleeding rate after colonic polypectomy [51] and in the treatment of itching skin conditions such as eczema [52]. 6\% Polidocanol Sclerotherapy is injected into placental implantation to reduce uterine bleeding [53].

\section{Side-Effects and Complications}

Complications and side-effects of foam Sclerotherapy remain uncommon. However, serious side effects and risks include transient neurologic issues, deep vein thrombosis and tissue necrosis [54]. Visual troubles are definitely more frequent with foam than with liquid [55]. Their clinical presentation is similar to 
the aura of a migraine, to some extent, transient neurological symptoms can be part of a migrainous syndrome. Strokes, transient ischemic attacks and palsies after injections of sclerosing foam are also been reported [56] [57]. The phenomenon of thrombus' extension or progression to the deep system, or migration, is always considered abnormal [58]. The overall incidence of thrombo-embolic events is finally lower after foam Sclerotherapy than after surgery [59]. Complete duplex check of the deep and muscular venous systems in case of any abnormal symptomatology after foam injections is necessary. Case analysis demonstrates that early treatment (usually with low molecular weight heparin and compression) brings about a constant recovery. Polidocanol leaked into the artery, induced extensive embolism, could cause inevitable necrosis [60]. At present, we can use radiographic or ultrasonic guidance during Sclerotherapy, it helps doctors acquire detailed information of any abnormal vasculature and the draining veins and adjacent arteries, an appropriate volume of sclerosant to prevent complications can be determined.

\section{Conclusion}

The safety and effectiveness of the clinical application of Polidocanol in the treatment of varicose veins, esophageal variceal, angioma, vascular Malformation, hemorrhoids, cystic diseases and chronic tendinitis have been verified by evidence-based medicine. Learn the technique and remember that about foam knowledge, understanding of the disease and complications, skill and training, allows us to implement a careful approach to this treatment. At present, on the basis of basic experiments and clinical research, the application of new indications has been carried out, and clinical data and clinical cases with reference significance have been accumulated. In the future, with the increasingly mature application of Polidocanol in clinical practice, the application scope will be further expanded and the application prospect will be broad.

\section{Conflicts of Interest}

The authors declare no conflicts of interest regarding the publication of this paper.

\section{References}

[1] Malik, A.S., Boyko, O., Atkar, N. and Young, W.F. (2001) A Comparative Study of MR Imaging Profile of Titanium Pedicle Screws. http://onlinelibrary.wiley.com/doi/10.1034/j.1600-0455.2001.042002291.x/pdf

[2] Eckmann, D.M., Kobayashi, S. and Li, M. (2005) Microvascular Embolization Following Polidocanol Microfoam Sclerosant Administration. Dermatologic Surgery, 31, 636-643. https://doi.org/10.1097/00042728-200506000-00004

[3] Parsi, K., Exner, T., Connor, D.E., et al. (2008) The Lytic Effects of Detergent Sclerosants on Erythrocytes, Platelets, Endothelial Cells and Microparticles Are Attenuated by Albumin and Other Plasma Components in Vitro. European Journal of Vascular and Endovascular Surgery, 36, 216-223. 
https://doi.org/10.1016/j.ejvs.2008.03.001

[4] Hamel-Desnos, C., Desnos, P., Wollmann, J.C., et al. (2003) Evaluation of the Efficacy of Polidocanol in the Form of Foam Compared with Liquid Form in Sclerotherapy of the Greater Saphenous Vein: Initial Results. Dermatologic Surgery, 29, 1170-1175.

[5] Eckmann, D.M. (2009) Polidocanol for Endovenous Microfoam Sclerosant Therapy. Expert Opinion on Investigational Drugs, 18, 1919-1927. https://doi.org/10.1517/13543780903376163

[6] Grosse-Siestrup, C., Unger, V., Pfeffer, J., et al. (2004) Hepatotoxic Effects of Polidocanol in a Model of Autologously Perfused Porcine Livers. Archives of Toxicology, 78, 697-705. https://doi.org/10.1007/s00204-004-0587-7

[7] Weiss, R.A., Feied, C.F. and Weiss, M.A. (2001) Vein Diagnosis and Treatment: A Comprehensive Approach. MaGrawHill, New York, 16.

[8] Tessari, L., Cavezzi, A. and Frullini, A. (2001) Preliminary Experience with a New Sclerosing Foam in the Treatment of Varicose Veins. Dermatologic Surgery, 27, 58-60.

[9] Eckmann, D.M., Kobayashi, S. and Li, M. (2005) Microvascular Embolization Following Polidocanol Microfoam Sclerosant Administration. Dermatologic Surgery, 31, 636-643. https://doi.org/10.1097/00042728-200506000-00004

[10] Nastasa, V., Samaras, K., Ampatzidis, C., et al. (2015) Properties of Polidocanol Foam in View of Its Use in Sclerotherapy. International Journal of Pharmaceutics, 478, 588-596. https://doi.org/10.1016/j.ijpharm.2014.11.056

[11] Guex, J.-J. (2005) Guex Foam Sclerotherapy: An Overview of Use for Primary Venous Insufficiency. Seminars in Vascular Surgery, 18, 225-294.

https://doi.org/10.1053/j.semvascsurg.2004.12.008

[12] Kostoglou, M., Georgiou, E. and Karapantsios, T.D. (2011) A New Device for Assessing Film Stability in Foams: Experiment and Theory. Colloids and Surfaces A: Physicochemical and Engineering Aspects, 382, 64-73. https://doi.org/10.1016/j.colsurfa.2010.12.028

[13] Schwartz, L.W. and Roy, R.V. (2003) A Mathematical Model for an Expanding Foam. Journal of Colloid and Interface Science, 264, 237-249. https://doi.org/10.1016/S0021-9797(03)00425-9

[14] Cameron, E., Chen, T., Connor, D.E., et al. (2013) Sclerosant Foam Structure and Stability Is Strongly Influenced by Liquid Air Fraction. European Journal of Vascular and Endovascular Surgery, 46, 488-494. https://doi.org/10.1016/j.ejvs.2013.07.013

[15] Valenzuela, G.C., Wong, K., Connor, D.E., et al. (2013) Foam Sclerosantsare More Stable at Lower Temperatures. European Journal of Vascular and Endovascular Surgery, 46, 593-599. https://doi.org/10.1016/j.ejvs.2013.08.012

[16] Varithena Delivery System: Instructions for Use. Provensis Ltd.

[17] Sta Maria, N. and Eckmann, D.M. (2003) Model Predictions of Gas Embolism Growth and Reabsorption during Xenon Anesthesia. Anesthesiology, 99, 638-645. https://doi.org/10.1097/00000542-200309000-00019

[18] Criqui, M.H., Jamosmos, M., Fronek, A., et al. (2003) Chronic Venous Disease in an Ethnically Diverse Population: The San Diego Population Study. American Journal of Epidemiology, 158, 448-456. https://doi.org/10.1093/aje/kwg166

[19] Kundu, S., Lurie, F., Millward, S.F., et al. (2007) Recommended Reporting Standards for Endovenous Ablation for the Treatment of Venous Insufficiency: Joint 
Statement of the American Venous Forum and the Society of Interventional Radiology. Journal of Vascular Surgery, 46, 582-589. https://doi.org/10.1016/j.jvs.2007.05.025

[20] Guex, J.J. (2005) Foam Sclerotherapy: An Overview of Use for Primary Venous Insufficiency. Seminars in Vascular Surgery, 18, 25-29. https://doi.org/10.1053/j.semvascsurg.2004.12.008

[21] De-Abreu, G.C.G., Júnior, C.O., de-Abreu, M.F.M., et al. (2017) Ultrasound-Guided Foam Sclerotherapy for Severe Chronic Venous Insufficiency. Revista do Colégio Brasileiro de Cirurgióes, 44, 511-520. https://doi.org/10.1590/0100-69912017005014

[22] Rabe, E., Schliephake, D., Otto, J., et al. (2010) Sclerotherapy of Telangiectases and Reticular Veins: A Double-Blind, Randomized, Comparative Clinical Trial of Polidocanol, Sodium Tetradecyl Sulphate and Isotonic Saline (EASI Study). Phlebology, 25, 124-131. https://doi.org/10.1258/phleb.2009.009043

[23] Vähäaho, S., Halmesmäki, K., Albäck, A., et al. (2018) Five-Year Follow-Up of a Randomized Clinical Trial Comparing Open Surgery, Foam Sclerotherapy and Endovenous Laser Ablation for Great Saphenous Varicose Veins. British Journal of Surgery, 105, 686-691. https://doi.org/10.1002/bjs.10757

[24] Schuller-Petrović, S., Pavlović, M.D., Neuhold, N., et al. (2011) Subcutaneous Injection of Liquid and Foamed Polidocanol: Extravasation Is Not Responsible for Skin Necrosis during Reticular and Spider Vein Sclerotherapy. Journal of the European Academy of Dermatology and Venereology, 25, 983-986. https://doi.org/10.1111/j.1468-3083.2010.03873.x

[25] Kim, Y.D. (2014) Management of Acute Variceal Bleeding. Clinical Endoscopy, 47, 308-314. https://doi.org/10.5946/ce.2014.47.4.308

[26] Ali, S.M., Wu, S., Xu, H., Liu, H., Hao, J. and Qin, C. (2017) A Prospective Study of Endoscopic Injection Sclerotherapy and Endoscopic Variceal Ligation in the Treatment of Esophageal Varices. Journal of Laparoendoscopic \& Advanced Surgical Techniques, 27, 333-341.

[27] Iwase, H., Shimada, M., Hirashima, N., et al. (2014) Long-Term Results of Radiologically Guided Endoscopic Injection Sclerotherapy for Esophageal Variceal Bleeding: A Retrospective 30-Year Survey. Journal of Gastrointestinal and Digestive System, 4, 238. https://doi.org/10.4172/2161-069X.1000238

[28] Dai, C., Liu, W.X., Jiang, M., et al. (2015) Endoscopicvariceal Ligation Compared with Endoscopic Injection Sclerotherapy for Treatment of Esophageal Variceal Hemorrhage: A Meta-Analysis. World Journal of Gastroenterology, 21, 2534-2541. https://doi.org/10.3748/wjg.v21.i8.2534

[29] Bao, H.W., Zhao, Q.-Y., Chen, F., Li, C.-X. and Jiang, T.-A. (2015) Clinical Efficacy of Lauromacrogol Percutaneous Intratumor Injection for Treatment of Liver Hemangioma. Chinese Journal of Interventional Imaging and Therapy, 12, 39-42.

[30] Yamaki, T., Nozaki, M., Sakurai, H., Takeuchi, M., Soejima, K. and Kono, T. (2008) Prospective Randomized Efficacy of Ultrasound-Guided foam Sclerotherapy Compared with Ultrasound-Guided Liquid Sclerotherapy in the Treatment of Symptomatic Venous Malformations. Journal of Vascular Surgery, 47, 578-584. https://doi.org/10.1016/j.jvs.2007.11.026

[31] Gao, Z., Zhang, Y., Li, W., et al. (2018) Effectiveness and Safety of Polidocanol for the Treatment of Hemangiomas and Vascular Malformations: A Meta-Analysis. Dermatologic Therapy, 31.

[32] Rabe, E., Breu, F.X. and Cavezzi, A. (2013) European Guidelines for Sclerotherapy in Chronic Venous Disorders. Phlebology, 29, 338-354. 
[33] Blaise, S., Charavin-Cocuzza, M., Riom, H., et al. (2011) Treatment of Low-Flow Vascular Malformations by Ultrasound-Guided Sclerotherapy with Polidocanol Foam: 24 Cases and Literature Review. European Journal of Vascular and Endovascular Surgery, 41, 412-417. https://doi.org/10.1016/j.ejvs.2010.10.009

[34] Al-Ghnaniem, R., Leather, A.J. and Rennie, J.A. (2001) Survey of Methods of Treatment of Haemorrhoids and Complications of Injection Sclerotherapy. Annals of the Royal College of Surgeons of England, 83, 325-328.

[35] Akerud, L. (1995) Sclerotherapy of Haemorrhoids: A Prospective Randomised Trial of Polidocanol and Phenol in Oil. Coloproctology, 17, 73-86.

[36] Fox, A., Tietze, P.H. and Ramakrishnan, K. (2014) Anorectal Conditions: Hemorrhoids. FP Essentials, 419, 11-19.

[37] Zellweger, U., Meyenberger, C., Bühler, H. and Ammann, R. (1990) Ultrasonically-Guided Sclerosing of Kidney and Liver Cysts Using Polidocanol. Schweizerische Rundschau fur Medizin Praxis, 79, 1412-1415.

[38] Goktay, A.Y., Secil, M., Ozcan, M.A. and Dicle, O. (2006) Percutaneous Treatment of Congenital Splenic Cysts: Drainage and Sclerotherapy with Polidocanol. CardioVascular and Interventional Radiology, 29, 469-472. https://doi.org/10.1007/s00270-004-0194-2

[39] Chen, Z.J., Wang, L., Huang, Y.J., Lai, S.Q., Chen, H.M., Pei, J.H., Cong, S.Z. and Kuang, J. (2016) Efficacy of Ultrasound-Guided Lauromacrogol Sclerotherapy for Benign Thyroid Cysts and Factors Affecting the Therapeutic Effect. Journal of Southern Medical University, 36, 1694-1699.

[40] Ormeci, N., Idilman, R., Akyar, S., Palabiyikoğlu, M., Coban, S., Erdem, H. and Ekiz, F. (2007) Hydatid Cysts in Muscle: A Modified Percutaneous Treatment Approach. International Journal of Infectious Diseases, 11, 204-208. https://doi.org/10.1016/j.ijid.2005.10.015

[41] Rastogi, S., Varshney, M.K., Trikha, V., Khan, S.A., Choudhury, B. and Safaya, R. (2006) Treatment of Aneurysmal Bone Cysts with Percutaneous Sclerotherapy Using Polidocanol. A Review of 72 Cases with Long-Term Follow-Up. The Bone \& Joint Journal, 88, 1212-1216. https://doi.org/10.1302/0301-620X.88B9.17829

[42] Yamaki, T., Sasaki, Y., Hasegawa, Y., Osada, A., Konoeda, H., Hamahata, A., Nozaki, M. and Sakurai, H. (2017) Percutaneous Ultrasound-Guided Sclerotherapy with Polidocanolmicrofoam for Lymphatic Malformations. Journal of Vascular Surgery: Venous and Lymphatic Disorders, 5, 707-714. https://doi.org/10.1016/j.jvsv.2017.03.017

[43] Alfredson, H., Ohberg, L. and Forsgren, S. (2003) Is Vasculo-Neural Ingrowth the Cause of Pain in Chronic Achilles tendinosis? An Investigation Using Ultrasonography and Colour Doppler, Immunohistochemistry, and Diagnostic Injections. Knee Surgery, Sports Traumatology, Arthroscopy, 11, 334-338. https://doi.org/10.1007/s00167-003-0391-6

[44] Alfredson, H. (2004) Chronic Tendon Pain-Implications for Treatment: An Update. Current Drug Targets, 5, 407-410. https://doi.org/10.2174/1389450043345353

[45] Fenelon, C., Galbraith, J.G., Hession, P. and D’Souza, L.G. (2017) Complete Tendon Achilles Rupture Following Injection of Aethoxysklerol (Polidocanol) for the Treatment of Chronic Achilles Tendinopathy. Foot and Ankle Surgery, 23, e7-e8. https://doi.org/10.1016/j.fas.2016.11.005

[46] Zeisig, E., Ohberg, L. and Alfredson, H. (2006) Sclerosing Polidocanol Injections in Chronic Painful Tennis Elbow-Promising Results in a Pilot Study. Knee Surgery, Sports Traumatology, Arthroscopy, 14, 1218-1224. 
https://doi.org/10.1007/s00167-006-0156-0

[47] Krogh, T.P., Bartels, E.M., Ellingsen, T., et al. (2013) Comparative Effectiveness of Injection Therapies in Lateral Epicondylitis: A Systematic Review and Network Meta-Analysis of Randomized Controlled Trials. The American Journal of Sports Medicine, 41, 1435-1446. https://doi.org/10.1177/0363546512458237

[48] Alfredson, H., Harstad, H., Haugen, S. and Ohberg, L. (2006) Sclerosing Polidocanol Injections to Treat Chronic Painful Shoulder Impingement Syndrome-Results of a Two-Centre Collaborative Pilot Study. Knee Surgery, Sports Traumatology, Arthroscopy, 14, 1321-1326. https://doi.org/10.1007/s00167-006-0205-8

[49] Kaneko, T., Nakao, A. and Takagi, H. (2000) Clinical Studies of New Material for Portal Vein Embolization: Comparison of Embolic Effect with Different Agents. Hepatogastroenterology, 47, 790-794.

[50] Guo, J.X., Lucchesi, L. and Gregory, K.W. (2015) Improvement of Stability of Polidocanol Foam for Nonsurgical Permanent Contraception. Contraception, 92, 103-107. https://doi.org/10.1016/j.contraception.2015.06.004

[51] Facciorusso, A., Di Maso, M., Antonino, M., et al. (2015) Polidocanol Injection Decreases the Bleeding Rate after Colon Polypectomy: A Propensity Score Analysis. Gastrointestinal Endoscopy, 82, 350-358.e2. https://doi.org/10.1016/j.gie.2015.01.003

[52] Hawro, T., Fluhr, J.W., Mengeaud, V., et al. (2014) Polidocanol Inhibits Cowhage-But Not Histamine-Induced Itch in Humans. Experimental Dermatology, 23, 922-923. https://doi.org/10.1111/exd.12555

[53] Malagón Reyes, R.M., Castorena de Ávila, R., Ángeles Vázquez, M.J., et al. (2016) Sclerotherapy with 6\% Polidocanol Solution in Patients with Placenta Accreta. Taiwanese Journal of Obstetrics \& Gynecology, 55, 654-658. https://doi.org/10.1016/j.tjog.2015.11.001

[54] Guex, J.J. (2009) Complications and Side-Effects of Foam Sclerotherapy. Phlebology, 24, 270-274. https://doi.org/10.1258/phleb.2009.009049

[55] Guex, J.-J., Allaert, F.-A., Gillet, J.-L. and Chleir, F. (2005) Immediate and Midterm Complications of Sclerotherapy. Report of a Prospective Multicenter Registry of 12173 Sclerotherapy Sessions. Dermatologic Surgery, 31, 123-128. https://doi.org/10.1097/00042728-200502000-00001

[56] Forlee, M.V., Grouden, M., Moore, D.J. and Shanik, G. (2006) Stroke after Varicose Vein foam Injection Sclerotherapy. Journal of Vascular Surgery, 43, 162-164. https://doi.org/10.1016/j.jvs.2005.09.032

[57] Busch, R.G., Derrick, M. and Manjoney, D. (2008) Major Neurological Events Following Foam Sclerotherapy. Phlebology, 23, 189-192. https://doi.org/10.1258/phleb.2007.007073

[58] Bergan, J., Pascarella, L. and Mekenas, L. (2006) Venous Disorders: Treatment with Sclerosant Foam. The Journal of Cardiovascular Surgery, 47, 9-18.

[59] Gillet, J.L., Guedes, J.M., Guex, J.J., et al. (2009) Side Effects and Complications of Foam Sclerotherapy of the Great and Small Saphenous Veins: A Controlled Multicentre Prospective Study including 1025 Patients. Phlebology, 24, 131-138. https://doi.org/10.1258/phleb.2008.008063

[60] Thomas, P., Martin, J. and Braithwaite, B. (2016) Case Report: Inadvertent Intra-Arterial Injection during Sclerotherapy May Not Be the Disaster You Think. Journal of Vascular Nursing, 34, 12-16. https://doi.org/10.1016/j.jvn.2015.09.003 